\title{
Neuroimaging in Epilepsy: Towards Structural Cellular Imaging
}

\author{
Ruben Kuzniecky
}

The evolution of neuroimaging over the past 20 years has had a remarkable impact in neurological and neurosurgical patient care $^{1-5}$. This impact has not been less important in epilepsy where the shift from a pure electrophysiological approach to an etiological one has occurred based largely on imaging findings ${ }^{6-9}$. Unlike in many other disorders, once a patient is given the diagnosis of epilepsy, it is important to define the syndrome underlying epilepsy. If it is focal in origin and not one of the benign variants, it is of utmost importance to try to identify a focal lesion causing or associated with the epilepsy. Unfortunately, the vast majority of patients with new onset localization related epilepsy do not have a clear imaging abnormality even though $30-40 \%$ of them will develop intractable epilepsy over time ${ }^{10}$. Therefore, it is extremely important to try to identify these lesions early on in the course of the disorder, since the prognosis of epilepsy often is based on the underlying etiology.

This review summarizes the current clinical use of structural MRI imaging based in epilepsy and discusses future developments and their impact in epilepsy.

\section{Imaging in Epilepsy}

Neuroimaging is more than simply the detection of lesions potentially associated with epilepsy. Although the etiological basis for some epilepsy conditions can be established with imaging such as in mesial temporal lobe sclerosis (MTS) or in malformations of cortical development (MCD), it is also important to remember that imaging can offer much more than just a simple representation of abnormal brain structure. Neuroimaging in conjunction with the clinical phenotype and other data may provide a novel syndromic classification in a given patient and may change management and prognosis. In addition, new developments such as functional imaging techniques may help us define with more exactitude the limits of the epileptogenic network and the interactions between these areas and functional cortex ${ }^{11,12}$. The importance of neuroimaging in the understanding of the developmental aspect of brain function and brain development can't be overemphasized in this context. Imaging can also be used as a predictive tool in epilepsy. This has been well established in MTS in patients undergoing epilepsy surgery. Numerous studies suggest that MTS is highly predictive of surgical success following temporal lobe epilepsy surgery while the absence of MTS correlates with a $50 \%$ probability of failure ${ }^{13-15}$.

The majority of patients with temporal lobe epilepsy may be defined from a traditional electrophysiologic perspective as having either neocortical or mesial temporal lobe epilepsy. This classification certainly limits our understanding of temporal lobe epilepsy, as the etiological basis is vastly heterogeneous and more complex. Nonetheless, at least in mesial temporal lobe epilepsy it is possible to sub define certain conditions. For example, the presence of MTS exclusively without any other evidence of adjacent structural atrophy can be classified as a pure MTS. When there is other evidence of pathology such as neocortical atrophy or temporal pole white matter changes, then the condition is classified as MTS plus. In contrast, the vast majority of patients with neocortical temporal lobe epilepsy have normal structural imaging studies and no clear classification scheme exist. In this group, better imaging techniques are needed to understand this condition.

In extra-temporal lobe epilepsy the problem is even more acute. The vast majority of patients with neocortical epilepsy have normal imaging studies. Several investigations over the past decade have consistently demonstrated that over $70 \%$ of patients with neocortical epilepsy have normal imaging studies $^{16,17}$. This raises major challenges to the management of these patients. Therefore, improving current techniques is important and developing new techniques will be crucial in increasing our diagnostic yield.

\section{Improving Diagnostic Yield}

The evaluation of patients with epilepsy is generally demanding, as many diagnostic techniques are simply inadequate to establish an etiological basis. We can improve the yield of MRI in epilepsy by manipulating different variables. These include better signal to noise ratio, improved white/grey matter contrast, higher in-plane resolution, statistically based structural analysis, co-registration of multi- imaging modalities and high field magnets.

\section{Improving Image Contrast}

Improving image contrast is primarily based on improving hardware and software technology. Over the past years, image averaging as well as higher resolution coils have improved image resolution. Image averaging is simply the summation of imaging data that are then co-registered off-line and analyzed $^{18,19}$. This manipulation improves white/gray matter contrast and improves relatively well the detection of certain lesions. The development of new head coils including those with 32-96-channel receivers has improved the diagnostic capability of current hardware technology. 32 channels arrays can increase

\footnotetext{
From the NYU Comprehensive Epilepsy Center, NYU University, New York, New York, USA.

Received September 7, 2012. Final Revisions Submitted September 7, 2012. Correspondence to: Ruben Kuzniecky, NYU Comprehensive Epilepsy Center, NYU University, New York, New York, USA. Email: Ruben.Kuzniecky@nyumc.org.
} 


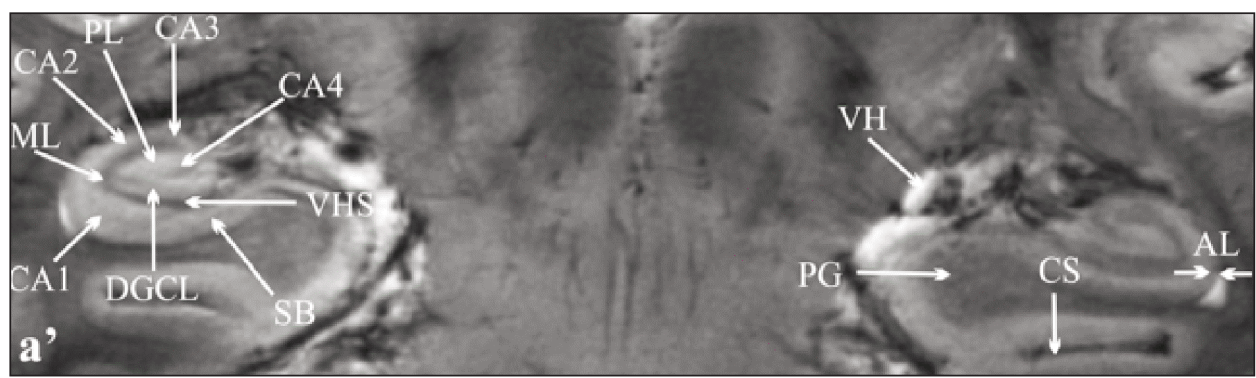

Figure 1: Hippocampal anatomy at 7T. Note the internal definition of the hippocampal C1-C4 sections.

SNR of about $8-12 \%$ while higher coils may increase SNR higher offering improved and faster imaging ${ }^{20-22}$.

The use of high field magnets has improved slightly the yield for identification of focal lesions. A number of studies have demonstrated that $3 \mathrm{~T}$ imaging is slightly superior to $1.5 \mathrm{~T}$ imaging in patients with epilepsy ${ }^{23-25}$. It is also very clear that $3 \mathrm{~T}$ offers several advantages over $1.5 \mathrm{~T}$ for functional imaging studies but those will not be discussed here.

Higher field magnets such as 4 to $7 \mathrm{~T}$ have begun to be explored to improve diagnostic yield in epilepsy ${ }^{26,27}$. Figure 1 shows the anatomical structure from a 7T study over normal hippocampus in humans (NYU imaging center). This has been applied preliminarily to patients with hippocampal pathology with a higher degree of morphological structural details with respect to the detection of these lesions. Of course the problem is that this high-field magnets remain a research tool and the cost of this unit will make it difficult for clinical applications at the present time. insufficient to detect subtle abnormalities. Over the past years, investigators have used different techniques to improve the sensitivity and specificity of lesion detection by both volumetric as well as other quantitative techniques. Statistical voxel morphometry can generate statistical maps of gray and white matter compared to normal brains. Many studies have shown that MCD and other lesions can be detected with this technique. In addition, a number of studies have shown that one can derive statistical maps of cortical thickness using this technology to detect areas of focal cortical thickening that are common in patients with focal cortical dysplasia and other malformations of brain development (Figure 2).

\section{Future Developments}

Functional MRI studies, in particular those using fMRI and functional connectivity are beginning to shed some interesting

\section{Image Processing Techniques}

In spite of large improvements in hardware, surface coils and scanning sequences, visual analysis of 2-D images are often

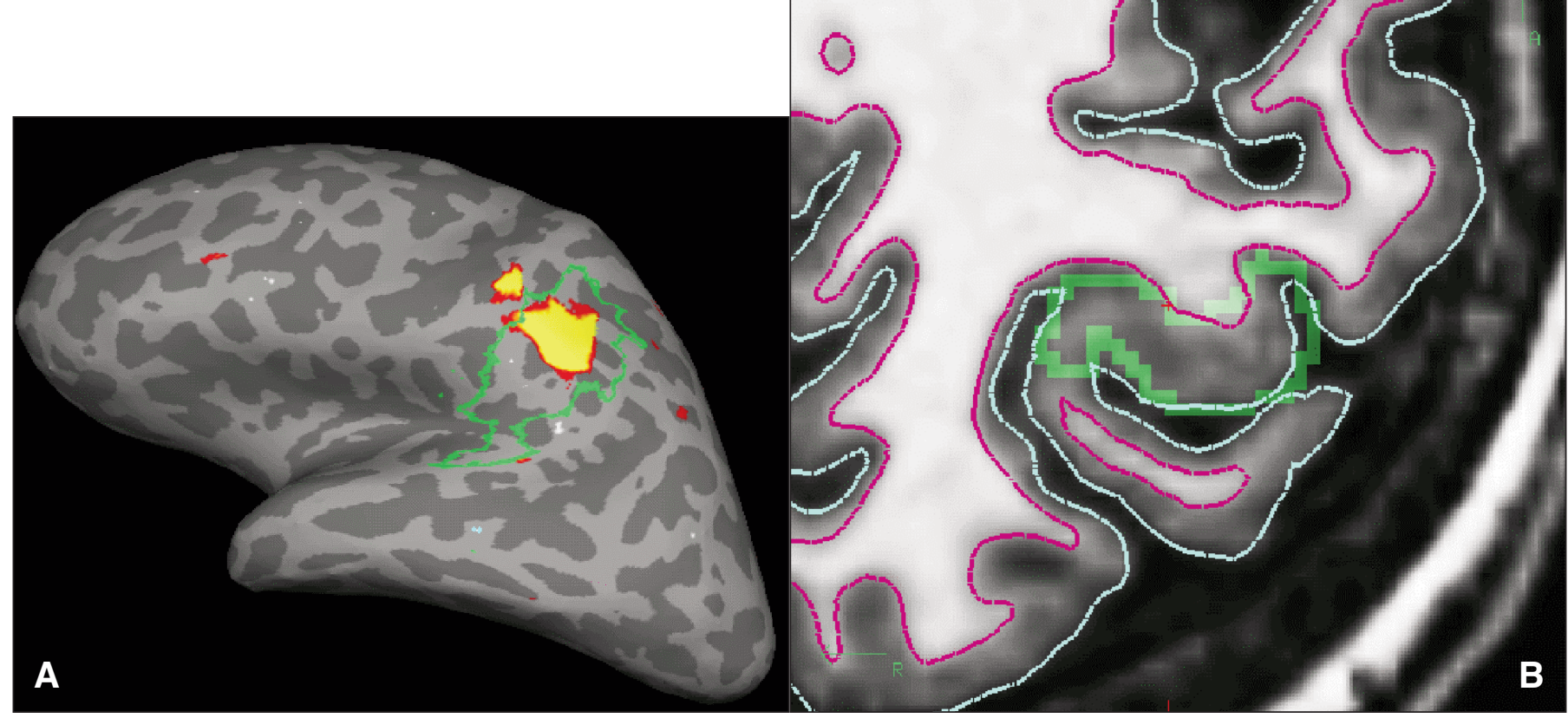

Figure 2: Free surfaser cortical thickness analysis. The statistically significant areas are shown as abnormal areas of cortical thickness with a Z-score of 10.8 compared to normal bran thickness. This area corresponded to an area of focal cortical dysplasia. 
information about the epileptic networks. A number of studies have shown for example that functional connectivity can be measured as activation in the resting state of functional networks that are temporally connected but spatially remote. These early studies are showing focal abnormalities in patients that have an epileptic area by showing decrease connectivity between cortical areas. Similarly, Zhang et al recently showed significant decoupling of functional and structural connectivity in generalized epilepsy. ${ }^{28}$

In summary, the detection of subtle lesions in patients with otherwise normal imaging studies has been clearly demonstrated using new technologies that uses sophisticated image analysis tools. The application of these techniques to study whole brain development in the context of epilepsy and use statistical models from group analysis to detect abnormalities of brain function is likely to help us define with better exactitude the area of potential epileptogenic areas. It is likely that over the next few years, we will be able to improve the resolution of these images to the degree that is hard to imagine today.

\section{REFERENCES}

1. Deoni SC. Magnetic resonance relaxation and quantitative measurement in the brain. Methods Mol Biol. 2011;711:65-108.

2. Filippi M, Rocca MA. MR imaging of multiple sclerosis. Radiology. 2011 Jun;259(3):659-81.

3. Alluri V, Toiviainen P, Jaaskelainen IP, Glerean E, Sams M, Brattico E. Large-scale brain networks emerge from dynamic processing of musical timbre, key and rhythm. Neuroimage. 2012 Feb 15;59 (4):3677-89.

4. Kubal WS. Updated imaging of traumatic brain injury. Radiol Clin North Am. 2012 Jan;50(1):15-41.

5. Langsjo JW, Alkire MT, Kaskinoro K, Hayama H, Maksimow A, Kaisti KK, et al. Returning from oblivion: imaging the neural core of consciousness. J Neurosci. 2012 Apr 4;32(14):4935-43.

6. Kuzniecky R, de la Sayette V, Ethier R, Melanson D, Andermann F, Berkovic $S$, et al. Magnetic resonance imaging in temporal lobe epilepsy: pathological correlations. Ann Neurol. 1987 Sep;22(3): 341-7.

7. Kuzniecky R, Cascino GD, Palmini A, et al. Structural imaging. In: Engel JJ, editor. Surgical treatment of the epilepsies - 2nd edition. New York: Raven Press; 1993. p. 197-200.

8. Kuzniecky RI. Neuroimaging of epilepsy: therapeutic implications. NeuroRx. 2005 Apr;2(2):384-93.

9. McDonald CR, Thesen T, Carlson C, Blumberg M, Girard HM, Trongnetrpunya $\mathrm{A}$, et al. Multimodal imaging of repetition priming: Using fMRI, MEG, and intracranial EEG to reveal spatiotemporal profiles of word processing. Neuroimage. 2010 Nov 1;53(2):707-17.

10. Kwan P, Brodie MJ. Early Identification of Refractory Epilepsy. N Engl J Med. 2000;342:314-9.

11. Wang Q, Sporns O, Burkhalter A. Network analysis of corticocortical connections reveals ventral and dorsal processing streams in mouse visual cortex. J Neurosci. 2012 Mar 28;32(13): 4386-99.

12. Pan JW, Spencer DD, Kuzniecky R, Duckrow RB, Hetherington H, Spencer SS. Metabolic networks in epilepsy by MR spectroscopic imaging. Acta Neurol Scand. 2012 May 11.

13. Chang EF, Quigg M, Oh MC, Dillon WP, Ward MM, Laxer KD, et al. Predictors of efficacy after stereotactic radiosurgery for medial temporal lobe epilepsy. Neurology. 2010 Jan 12;74(2): 165-72.

14. Berkovic SF, McIntosh AM, Kalnins RM, Jackson GD, Fabinyi GC, Brazenor GA, et al. Preoperative MRI predicts outcome of temporal lobectomy: an actuarial analysis. Neurology. 1995 Jul;45(7):1358-63.

15. Kuzniecky R, Burgard S, Faught E, Morawetz R, Bartolucci A. Predictive value of magnetic resonance imaging in temporal lobe epilepsy surgery. Arch Neurol. 1993 Jan;50(1):65-9.
16. Holt RL, Provenzale JM, Veerapandiyan A, Moon WJ, De Bellis MD, Leonard S, et al. Structural connectivity of the frontal lobe in children with drug-resistant partial epilepsy. Epilepsy Behav. 2011 May;21(1):65-70.

17. Widjaja E, Mahmoodabadi SZ, Snead OC, 3rd, Almehdar A, Smith ML. Widespread cortical thinning in children with frontal lobe epilepsy. Epilepsia. 2011 Sep;52(9):1685-91.

18. Lupo JM, Li Y, Hess CP, Nelson SJ. Advances in ultra-high field MRI for the clinical management of patients with brain tumors. Curr Opin Neurol. 2011 Dec;24(6):605-15.

19. Duyn JH, Koretsky AP. Novel frontiers in ultra-structural and molecular MRI of the brain. Curr Opin Neurol. 2011 Aug;24(4): 386-93.

20. Kim JH, Moon CH, Park BW, Furlan A, Zhao T, Bae KT. Multichannel transceiver dual-tuned RF coil for proton/sodium MR imaging of knee cartilage at 3 T. Magn Reson Imaging. 2012 May;30(4):562-71.

21. Gruschke OG, Baxan N, Clad L, Kratt K, von Elverfeldt D, Peter A, et al. Lab on a chip phased-array MR multi-platform analysis system. Lab Chip. 2012 Feb 7;12(3):495-502.

22. Bandettini PA, Bowtell R, Jezzard P, Turner R. Ultrahigh field systems and applications at $7 \mathrm{~T}$ and beyond: progress, pitfalls, and potential. Magn Reson Med. 2012 Feb;67(2):317-21.

23. Pro-Sistiaga P, Lamberton F, Boraud T, Saulnier R, Young AR, Bioulac B, et al. High resolution $3 \mathrm{~T}$ fMRI in anesthetized monkeys. J Neurosci Methods. 2012 Mar 30;205(1):86-95.

24. Strandberg M, Larsson EM, Backman S, Kallen K. Pre-surgical epilepsy evaluation using 3T MRI. Do surface coils provide additional information? Epileptic Disord. 2008 Jun;10(2):83-92.

25. Alvarez-Linera J.3T MRI: advances in brain imaging. Eur J Radiol. 2008 Sep;67(3):415-26.

26. Metcalf M, Xu D, Okuda DT, Carvajal L, Srinivasan R, Kelley DA, et al. High-resolution phased-array MRI of the human brain at 7 tesla: initial experience in multiple sclerosis patients. J Neuroimaging. 2010 Apr;20(2):141-7.

27. Kang CK, Park CA, Kim KN, Hong SM, Park CW, Kim YB, et al. Non-invasive visualization of basilar artery perforators with $7 \mathrm{~T}$ MR angiography. J Magn Reson Imaging. 2010 Sep;32(3): 544-50.

28. Zhang Z, Liao W, Chen H, Mantini D, Ding JR, Xu Q, et al. Altered functional-structural coupling of large-scale brain networks in idiopathic generalized epilepsy. Brain. 2011 Oct;134(Pt 10): 2912-28. 